\title{
Effect of Cementation Technique of Individually Formed Fiber-Reinforced Composite Post on Bond Strength and Microleakage
}

\author{
Dominika Makarewicz, Anna-Maria B. Le Bell-Rönnlöf*, Lippo V.J. Lassila and Pekka K. Vallittu \\ Department of Biomaterials Science, Institute of Dentistry and BioCity Turku Biomaterials Research Program, \\ University of Turku, FI-20520, Turku, Finland
}

\begin{abstract}
Objectives: The aim of this study was to evaluate the effect of two different cementation techniques of individually formed E-glass fiber-reinforced composite (FRC) post on bond strength and microleakage.

Methods: The crowns of extracted third molars were removed and post preparation was carried out with parapost drills (diameter $1.5 \mathrm{~mm}$ ). After application of bonding agents individually formed FRC posts (everStick POST, diameter 1.5 $\mathrm{mm}$ ) were cemented into the post spaces with either ParaCem ${ }^{\circledR} U$ niversal or self-adhesive RelyX ${ }^{\mathrm{TM}}$ Unicem, using two different cementation techniques: 1) an "indirect (traditional) technique" where the post was prepolymerized prior application of luting cement and insertion into the post space or 2) a "direct technique" where the uncured post was inserted to the post space with luting cement and light-polymerized in situ at the same time. After water storage of 48 hours, the roots ( $n=10$ /group) were cut into discs of thickness of $2 \mathrm{~mm}$. A push-out force was applied until specimen fracture or loosening of the post. A microleakage test was carried out on roots which were not subjected to the loading test $(n=32)$ to evaluate the sealing capacity of the post-canal interface. The microleakage was measured using dye penetration depth under a stereomicroscope.

Results: Higher bond strength values $(\mathrm{p}<0.05)$ and less microleakage $(\mathrm{p}<0.05)$ were obtained with the "direct technique" compared to the "indirect technique". None of the FRC posts revealed any dye penetration between the post and the cement.

Conclusions: The "direct technique" seems to be beneficial when cementing individually formed FRC posts.
\end{abstract}

Keywords: Bond strength, Cementation technique, Custom-made fiber post, Dye penetration, Fiber-reinforced composite root canal post, Individually formed fiber post, Microleakage, Push-out force.

\section{INTRODUCTION}

In the last decades, the continuous development in fiberreinforced composites (FRC) has extended the clinical applications of FRCs into many areas such as periodontal splinting, minimal invasive fixed partial dentures, removable dentures, orthodontic applications and post and core restorations [1-7]. Cast metal posts and cores are more and more being replaced by prefabricated posts $[8,9]$. Prefabricated posts are root canal posts that are ready-to-use as chairside material. They can be made from metal, glass fiber or carbon fiber and they come in variety of shapes and surface design. Because of the high root fracture risk with prefabricated metal posts, nowadays prefabricated FRC posts, with biomechanical advantages are preferred [7]. However, also prefabricated FRC posts have disadvantages. They require preparation of the root canal to fit the shape of the post, which causes loss of dentin and makes the root more vulnerable to root fracture [10]. Large root preparation can be avoided by using individually formed FRC posts, which were recently introduced

*Address correspondence to this author at the Department of Biomaterials Science, Institute of Dentistry and BioCity Turku Biomaterials Research Program, University of Turku, FI-20520 Turku, Finland;

Tel: +358 2333 8297; Fax: +358 2333 8390; E-mail: ami.lebell@utu.fi
[11-13]. An individually formed FRC post can be polymerized in situ in the root canal, thus precisely following the shape of the canal [14].

In situ polymerization of an individually formed FRC post enables free radical polymerization to occur between the resin matrix of the FRC post and the composite resin luting cement. An oxygen inhibition layer is formed on the coronal part of the post, which allows free radical polymerization between the post and core-built-up composite resin [15]. The polymer matrix of individually formed FRC post material consists of both linear and cross-linked phases, which is called semi-interpenetrating polymer network (IPN). This matrix also allow formation of secondary IPN bonding based on interdiffusion of the resin systems of post and luting cement [15-17]. The manufacturer's recommendation has been to light-polymerize in two phases; first a short curing is carried out when the post material is placed in the root canal to copy the anatomical shape of the canal. After that the final curing is carried out after removing the post from the canal, in order to ensure complete curing also at the apical parts of the post. The final cementation of the individually formed FRC post is carried out in the same way as with the conventional solid prefabricated FRC posts. However, in spite of the advantages of the minimal invasive preparation, the extra 
Table 1. Materials Used in this Study

\begin{tabular}{|c|c|c|c|}
\hline Trade name & Type & Composition & Manufacturer \\
\hline \hline Stick®RESIN & light-curing resin & Bis-GMA-TEGDMA & Stick Tech Ltd, Turku, Finland \\
\hline everStick®POST 1.5 & E-glass fiber-reinforced composite (FRC) & PMMA, BisGMA & Stick Tech Ltd, Turku, Finland \\
\hline ParaCem®Universal, Base shade: white & Dual-cured composite resin luting cement & Bis-GMA, bis-EMA, TEG-DMA & Coltene-Whaledent, USA \\
\hline RelyX ${ }^{\text {TMUnicem }}$ & Self-adhesive universal resin cement & $\begin{array}{c}\text { Bifunctional methacrylate, multi- } \\
\text { functional phosphoric acid, me- } \\
\text { tyhacrylates }\end{array}$ & 3 3M ESPE AG, Germany \\
\hline Pararosaniline chloride & staining liquid & basic parafuchsine & Sigma-Aldrich, Finland \\
\hline
\end{tabular}

clinical steps, when forming the uncured individual FRC post, can make the use of this post material clinically more complicated and may need simplification if important properties of bonding and cement sealing are not compromised.

Bonding of a FRC root canal post to a root depends on the adhesion of luting cement to the post and to the root canal dentin. Therefore, the aim of this study was to determine bonding properties of an individually formed FRC post polymerized together with luting cement directly in the root canal ("direct technique") and to compare to those of an individually formed prepolymerized FRC post cemented into the root canal using the instructed ("indirect") technique. The hypothesis of this study is that an individually formed FRC post cemented using the direct technique would result in similar bonding properties to the root canal than it would have been if cemented by the indirect technique.

\section{MATERIALS AND METHODOLOGY}

Seventy-two $(n=72)$ extracted intact and caries-free human third molars, were stored in chloramine $(0.5 \%)$ for one month. The crowns of the teeth were removed at the cemento-enamel junction by wet grinding ( $\mathrm{SiC}$ paper, grit 180 FEPA, Federation of European Producers of Abrasives) and post space preparation up to $5 \mathrm{~mm}$ (measured from the orifice) was carried out with parapost drills (diameter $1.6 \mathrm{~mm}$ ) to the root that had the largest diameter (Push-out test $n=40$ and Microleakage test $n=32$ ). The composition of the materials used in this study is presented in Table $\mathbf{1}$.

\section{The "Indirect Technique" for Cementation of an Indi- vidually Formed FRC Post}

Preimpregnated continuous unidirectional E-glass fiberreinforcement (everStick POST, Stick Tech Ltd, Turku, Finland) was cut to a premeasured length and inserted into the root canal $(n=20)$. The post was initially light-polymerized inside the root canal for $20 \mathrm{~s}$. A light-polymerizing device (Optilux 501, Danbury, USA) with a halogen lamp radiating blue light (wavelength ranging between 500 and $700 \mathrm{~nm}$ ) and with an intensity of $780 \mathrm{~mW} / \mathrm{cm}^{2}$ (Optilux 501, Danbury, USA) was used. After removing the post from the canal, it was further light-polymerized for a total of $40 \mathrm{~s}$. The surface of the post was activated for the formation of secondary IPN bonding using light-curable resin (Stick®Resin, Stick Tech Ltd, Turku, Finland) and the post was placed under a light shield for 3-5 min to prevent premature polymerization of the activation resin by light. Prior to cementation, the resin layer was thinned by carefully blowing the surface of the post with dry air. The post was light-polymerized for $10 \mathrm{~s}$.

\section{The "Direct Technique" for Cementation of an Individu-} ally Formed FRC Post

Preimpregnated continuous unidirectional E-glass fiberreinforcement (everStick POST, Stick Tech Ltd, Turku, Finland) was cut to a premeasured length and fitted into the root canal $(n=20)$. The obtained post shape was cylindrical, achieved by rolling on a glass plate before. In the "direct technique" the uncured individually formed FRC post was inserted directly into the root canal together with the luting cement and light-polymerized together.

For both groups (indirect and direct techniques) post cementation was carried out with two different luting cements ( $\mathrm{n}=10$ /group, 4 groups): 1) ParaCem Universal DC Cement, which is a dual-cure resin cement and 2) RelyX $\mathrm{X}^{\mathrm{TM}}$ Unicem, which is a dual-cure, self-adhesive resin cement. The cementation procedure was depending on the luting cement used: 1) the post space was washed with water thoroughly, dried with paper points and lightly air-dried. The ParaBond®NonRinse Conditioner (ParaCem ${ }^{\circledR}$ Universal, ColteneWhaledent, USA) was applied to the pretreated root canal with a brush and massaged for $30 \mathrm{~s}$. After removing excess Non-Rinse Conditioner from the root canal with a paper point and lightly air-drying, one drop of ParaBond ${ }^{\circledR}$ Adhesive A (ParaCem ${ }^{\circledR}$ Universal, Coltene-Whaledent, USA) was mixed with one drop of ParaBond ${ }^{\circledR}$ Adhesive B (ParaCem®Universal, Coltene-Whaledent, USA) and the mixed adhesive was applied to the root canal. The mixed adhesive was left in the root canal for $30 \mathrm{~s}$. Excess of adhesive was removed from the root canal with a paper point. Then the entire bond layer was lightly air-dried according to the manufacturer's instructions. The posts were cemented with dual-curing composite luting cement (ParaCem®Universal, Coltene-Whaledent, USA) into the prepared post spaces of the roots. The cement was light-cured (in $45^{\circ}$ angle, close to the root of the post) for $40 \mathrm{~s}$ (20 s on each side). After cementation, specimens were stored in water at a temperature of $37^{\circ} \mathrm{C}$ for 48 hours. 2) The post space was washed with water thoroughly, dried with paper points and lightly airdried. According to the manufacturer's instructions for use, bonding and conditioning of the prepared tooth structure is not necessary when using the self-adhesive cement (RelyX ${ }^{\mathrm{TM}}$ Unicem). The capsule containing cement was activated according to the instructions for use in the Aplicap ${ }^{\mathrm{TM}}$ Activator (3M ESPE). Then the capsule was mixed in a high-frequency mixer (Maxicap ${ }^{\mathrm{TM}}, 3 \mathrm{M}$ ESPE) for 


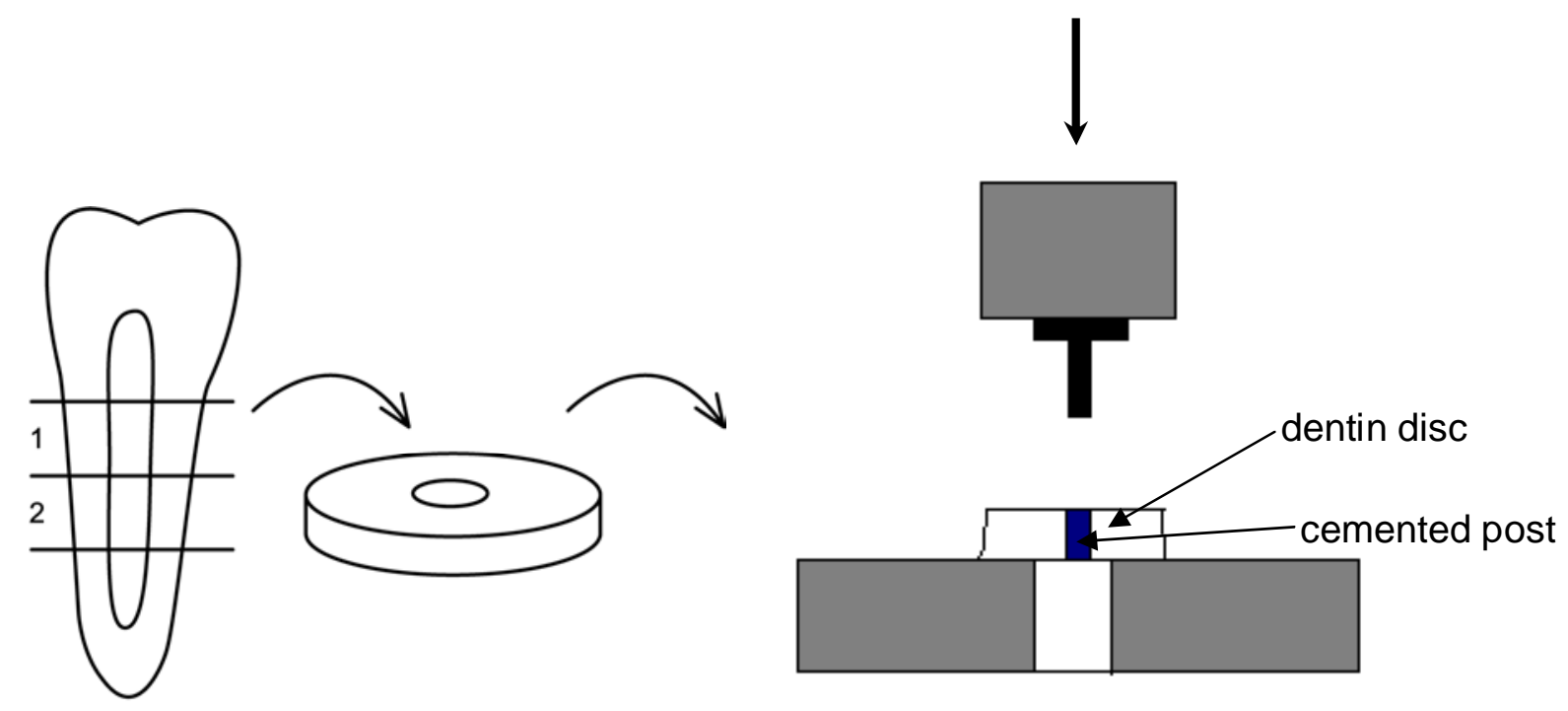

Fig. (1). A setup of the custom-made jig for measuring the push-out force (modified from Le Bell et al 2005 [24]). 1= coronal layer, 2= apical layer.

mixer (Maxicap ${ }^{\mathrm{TM}}$, 3M ESPE) for $15 \mathrm{~s}$. The capsules were inserted in the Aplicap, the cement was applied into the post spaces and the individually formed FRC post was positioned in the post space. The cement was light-polymerized (in $45^{\circ}$ angle, close to the root of the post) for $40 \mathrm{~s}(20 \mathrm{~s}$ from each side). After cementation, specimens were stored in water at a temperature of $37^{\circ} \mathrm{C}$ for 48 hours.

\section{Push-Out Test}

Four push-out test groups with 10 teeth each $(n=$ 10/group) were prepared. The coronal part of the root specimen were wet ground to flat (grit 180 FEPA) and the root specimen were cut with a low-speed diamond saw (Leitz 1600 Saw Microtome) into discs of thickness of $2 \mathrm{~mm}( \pm$ $0.20 \mathrm{~mm}$ ). The push-out force was measured by applying a force to the upper end of the post with a universal testing machine (Lloyd LRX, Lloyd Instruments Ltd, Fareham, UK) with a custom-made jig using a cross-head speed of 1.0 $\mathrm{mm} / \mathrm{min}$. Two discs of each root were measured (" $1{ }^{\text {st }}$ layer"= coronal disc, " 2 nd layer"= apical disc) $($ Fig. 1). The force at the point of interfacial failure between the post, cement and dentin was observed from the loading curve. The force (Newton) required to debond the post from the dentin disc was registered for all posts. The push-out force (MPa) was calculated from the formula

$$
\sigma=\mathrm{F} / 2 \pi \mathrm{r}
$$

where $\mathrm{F}$ is the debonding force $(\mathrm{N})$ and $\mathrm{r}$ is radius of post.

Failure modes were categorized as interfacial failure between the post-cement or post-dentin or mixed mode failure.

\section{Microleakage Test}

Four groups, each consisting of 8 teeth, were investigated in the microleakage test. The teeth $(n=32)$ were prepared in the same way as mentioned above. After post cementation, the acrylic resin embedding and sealing the tooth tightly was entirely coated with nail varnish to within $1 \mathrm{~mm}$ of the cement margins from the chamber surface.
After immersion in $0.5 \%$ basic parafuchsine dye solution ( 24 hours, $37^{\circ} \mathrm{C}$ ), the specimens ( $n=8$ /group) were finally rinsed under running tap water and the nail varnish was removed (SiC paper, grit 320 FEPA, Federation of European Producers of Abrasives) from all surfaces.

The teeth were sectioned with a low speed saw, vertically dividing the posts into halves. After sectioning the specimens' surfaces were smoothened (SiC paper, grit 4000 FEPA, Federation of European Producers of Abrasives). Dye penetration was measured using a stereomicroscope at $10 \mathrm{x}$ magnification. The sections/halves were then studied under a stereomicroscope to measure the depth of dye penetration between the cement and dentin (or post and cement) from the chamber surface (Leica DC Twain, Version: 5.1.10, Cambridge, UK).

The data was analyzed by 3-way ANOVA using independent factors (cement, cementation technique and layer). Interaction between factors was evaluated and Levene's test was carried out. The level of statistical significance was set at 0.05 .

\section{RESULTS}

The results of the push-out test are shown in Fig. (2). ANOVA revealed that all factors (cement, technique and layer) were statistically significant $(\mathrm{P}<0.05)$. No interaction between factors was found ( $p>0.1)$. The individually formed FRC posts polymerized together with the luting cement in the root canal ("direct technique") revealed highest push-out strength and the difference to that of the specimens cemented with the "indirect technique" was significant $(P=0.05)$.

Furthermore, higher bond strength values $(\mathrm{P}=0.025)$ were obtained using ordinary dual-cure cement (ParaCem ${ }^{\circledR}$ Universal) in comparison to self-adhesive dual-cure cement (RelyX ${ }^{\mathrm{TM}}$ Unicem). A trend of coronal discs $\left(1^{\text {st }}\right.$ layers) revealing slightly higher push-out strength than the apical discs $\left(2^{\text {nd }}\right.$ layers $)(P=0.04)$ was also seen. 


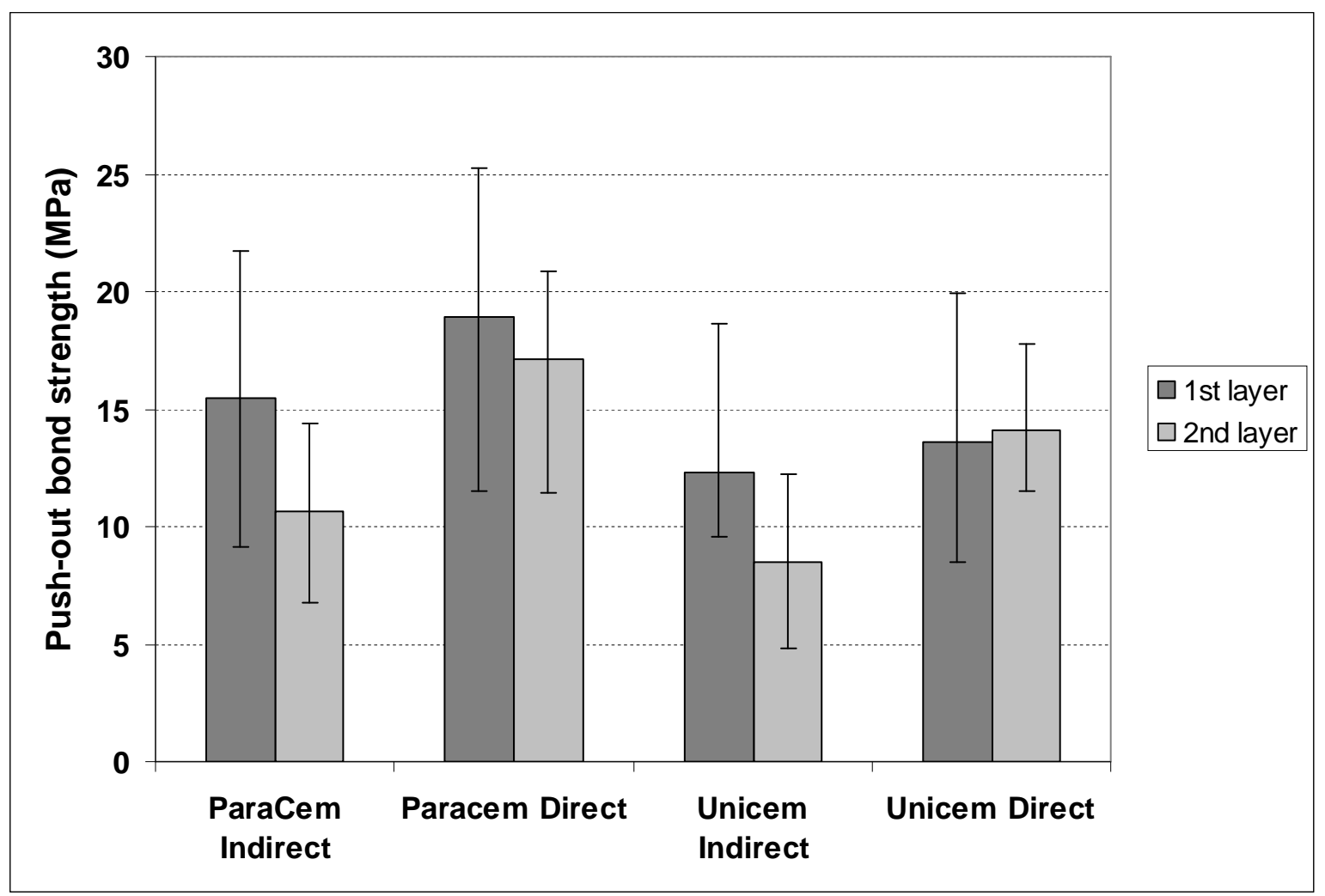

Fig. (2). Mean push-out bond strengths (MPa) and standard deviations (vertical lines) for individually formed FRC posts (everstick Post). Individually formed FRC posts were cemented either with the indirect technique or the direct technique with self-adhesive dual-cure cement (RelyX ${ }^{\mathrm{TM}}$ Unicem) and ordinary dual-cure cement (Paracem) for both types of cementation technique.

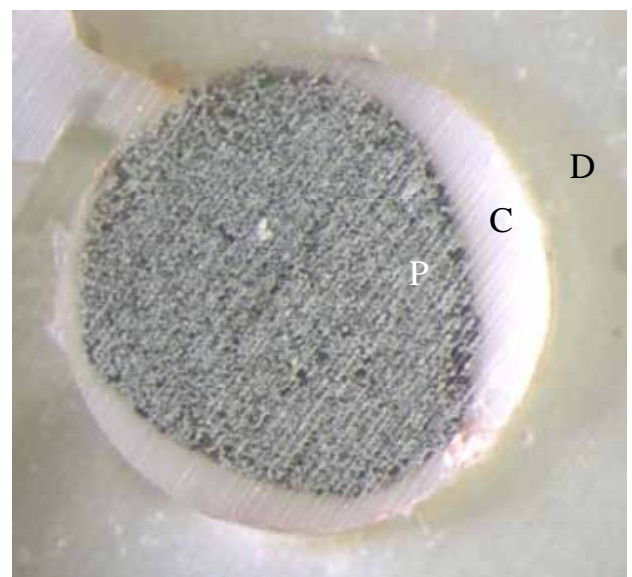

Fig. (3). Typical failure mode of the cemented posts after the push-out study: adhesive failure between cement and dentin of individually formed FRC post (everstick Post).

$\mathrm{D}=$ Dentine, $\mathrm{C}=$ Cement, $\mathrm{P}=$ Post.

In the assessment of the failure mode under a stereomicroscope it was found that none of the individually formed glass FRC posts showed adhesive failures between the post and the cement. The individually formed FRC posts in both groups ("direct" and "indirect" technique) failed mostly adhesively between the cement-dentin interface (Fig. 3). No difference in the failure types could be seen between the two groups.

The microleakage studies under a stereomicroscope revealed a significant difference in the depth of dye penetration between the posts cemented with the "direct technique" compared to those cemented with the "indirect technique" (Fig. 4 and 5). None of the individually formed FRC posts showed dye penetration between the post and the cement. Also dye penetration between the cement and the dentin was significantly decreased in the "direct technique" group. Furthermore, less microleakage was observed in the specimen where ordinary dual-cure cement (Paracem) had been used in comparison to the specimen which had been cemented with self-adhesive dual-cure cement (Rely $\mathrm{X}^{\mathrm{TM}}$ Unicem) (Fig. 4 and 5). 


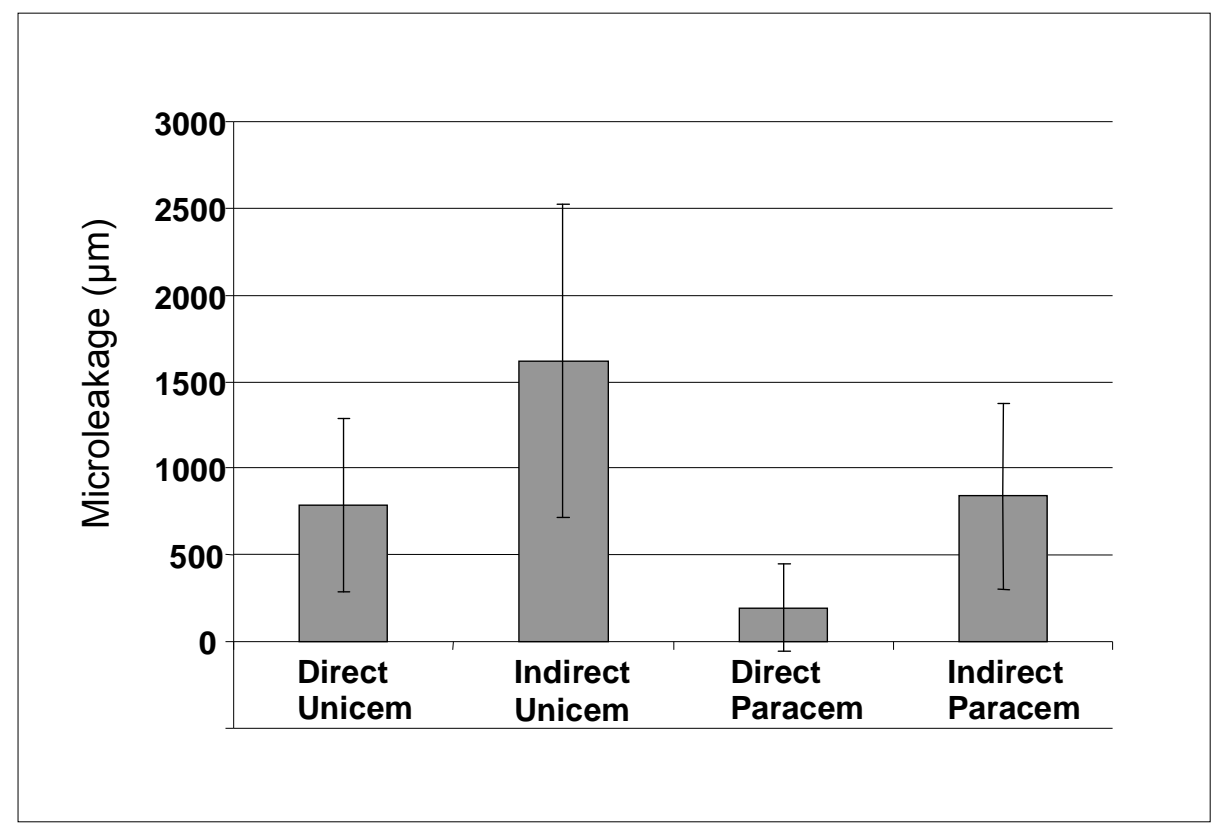

Fig. (4). Mean depth of dye penetration $(\mu \mathrm{m})$ along the dentin and cement interface and standard deviations (vertical lines) for individually formed FRC post (everstick Post) cemented with either the indirect technique or the direct technique with self-adhesive dual-cure cement (RelyX ${ }^{\mathrm{TM}}$ Unicem) and ordinary dual-cure cement (ParaCem®Universal) for both types of cementation technique.

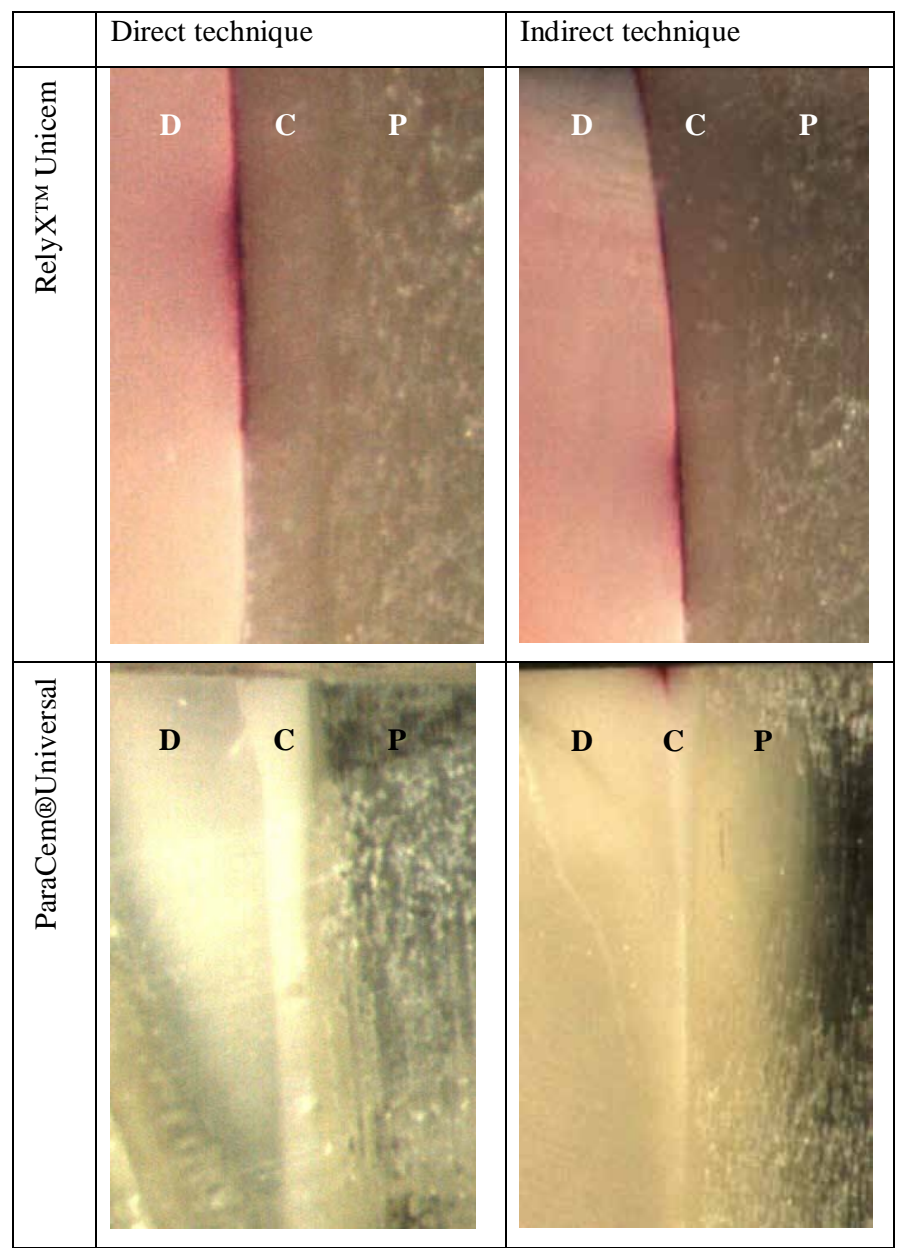

Fig. (5). The depth of dye penetration along the dentin and cement interface of individually formed FRC post (everstick Post) cemented with the two different cements (ParaCem ${ }^{\circledR}$ Universal and RelyX ${ }^{\mathrm{TM}}$ Unicem) with two different cementation techniques (direct and indirect). $\mathrm{D}=$ Dentine, $\mathrm{C}=$ Cement, $\mathrm{P}=$ Post. 


\section{DISCUSSION}

For a successful root canal anchored restoration a strong bond between the post, the resin luting cement and the dentin is needed. To evaluate and examine the different interfaces between post, resin luting cement and dentin, many methods in different studies have been used, e.g. push-and pull-out force, microtensile test, shear bond test, finite element method (FEM)-studies and observational studies using SEM images. In this study a push-out and a microleakage test was carried out to evaluate the effect of two different cementation techniques of individually formed E-glass fiber-reinforced composite (FRC) posts.

The use of FRC posts, with a modulus of elasticity close to that of dentin, has rapidly increased [7]. It has been shown in many studies that FRC posts are a viable alternative to cast and prefabricated metal posts [8]. However, prefabricated FRC posts also have limitations in their properties, such as poor anatomical fit to the canal, poor bonding to luting cement and in thin posts low fracture resistance, and therefore individually formed FRC posts have been introduced $[11,12,18]$. In the individually formed, also called custom-made FRC posts, the fiber volume at the coronal part of the root canal is high and it fills the entire available root canal space. This increases the stiffness and strength of that part of the post and forms strong support for the core. By considering the mechanics of tubular structure of tooth and post system, the individually formed posts also provide fiber location closer to the outermost surface of the root where the highest functional stresses are located. In the case of prefabricated conventional FRC posts, the location of the post in the center of root - in the neutral axis of tubular structure - is not optimal to provide effective reinforcing effect by the fibers of the post for the root-core-crown system. In addition, by using the prefabricated FRC posts, the free space of the coronal root canal opening is filled only with weaker particulate filler composite resin cement.

Prefabricated FRC posts have been critized for their highly cross-linked polymer matrix, which is difficult to bond to resin luting cements and core material [17, 19, 20]. This is due to the fact that the monomers of composite resin luting cements, which are used when bonding FRC posts, cannot dissolve, penetrate and swell the polymer matrix of a cross-linked character [15, 17, 20-22]. Attempts to develop alternative polymer matrices of FRC posts have been made and results of a multiphase polymer matrix, consisting of both linear and cross-linked polymer phases (semiinterpenetrationg polymer network, semi-IPN), have been promising [12, 20, 23, 24]. The monomers of the adhesive resins and cements can diffuse into the linear polymer phase, swell it, and by polymerization, form interdiffusion bonding and a so-called secondary semi-IPN structure $[15,16,17,25$, 26]. Improved bonding allows transfer of loads from the crown-core system to the root trough the root canal post.

Good adhesion between post and cement, and cement and dentin is one of the important factors in load transfer. Another is the load bearing capacity, i.e. resistance of the post itself against deformation by occlusal forces with different directions. Flexural strength of present FRC posts are ca. $1100 \mathrm{Mpa}$ [11]. Although it is known that strength values reported in MPa are influenced by diameter of the post versus length of the span in the test design, the ultimate loadbearing capacity is in relation to the diameter of the post [27]. The concept of using individually formed FRC posts is based on minimizing the preparation need to the deeper parts of the root canal, and allowing addition of higher quantity of FRC material to the coronal root canal opening of the tooth. Thus, the concept minimizes stress at the apical parts of the post and enables a stiff and fracture resistant post with larger diameter close to the core. The bonding of luting cement and core-built-up composite resin is ensured by secondary IPN mechanism.

When comparing the luting and curing methods of directly and indirectly made FRC posts, some important aspects need to be discussed. Firstly, it is known that unidirectional FRC is an anisotropic material, in which physical, thermal and optical properties vary along the fiber direction $[28,29]$. At polymerization stage, also the polymerization shrinkage vary, being low in the directions of fibers and higher in the perpendicular direction to the fibers $[30,31]$. This was assumed to cause gap formation to the dentinecement or cement-post interphase. This expectation was supported by the geometry of the root canal as a cavity where polymerization of the composite resin occurs: a root canal is a high $\mathrm{C}$-factor (cavity configuration factor) cavity in which the ratio of the restorations's bonded to unbonded free surfaces causes increased polymerization stress formation to the bonded cavity walls [32]. For instance, Class I and Class II cavities and flat surfaces, have mean C-factors of 4.03, 1.85 and $<0.20$, respectively [33]. The geometry of a root canal is similar to the geometry of a Class I cavity, although the root canal is deeper. The C-factor of a root canal is 953 [34]. Anisotropic polymerization contraction of the unidirectional FRC post, when it is polymerized directly in the root canal, increases the effect of a high $\mathrm{C}$-factor. Thus, it was assumed in this study that the indirectly made polymerization of the FRC post and the following cementation of the post would have caused less gap formation and therefore less dye penetration than for posts made directly in the root canal. However, the results were opposite: the directly made polymerization of the FRC post with both composite cements showed lower dye penetration and less gap formation between the cement-dentine interface.

One explanation could be that the polymerization of the resin in the root canal has not been complete which could have affected less polymerization shrinkage. However, earlier it has been demonstrated that the curing depth of FRC post material with appropriate degree of monomer conversion is $16 \mathrm{~mm}$, which exceeds the deepest portion of the post used in this study [14]. It has also been shown that scattering of light from FRC post made of E-glass is favourable for the curing of surrounding resins, such as luting cements [35]. In fact, by the start of polymerization reaction of methacrylate resins, the change of the refractive index of the resin systems improves even more light transmittance to the resin around the FRC post. Thus, there need to be some other explanation for the reduced gap formation.

Polymer matrix of the FRC post used in the present study is composed of cross-linking monomer system of bisphenolA-dimethacrylate (Bis-GMA) and linear polymers of po- 
lymethyl methacrylate (PMMA) which structurally form semi-interpenetrating polymer networks (semi-IPN). Resiliency of semi-IPN based composites is higher and modulus of elasticity is lower compared to polymer made of crosslinking monomers only $[36,37]$. This may have influenced the situation where the stress by the polymerization contraction remained the slightly plasticized polymer matrix of the FRC post rather than caused high debonding stresses at the dentine-cement-post interfaces. It is also possible, that the lower polymerization rate of the semi-IPN resin systems allows the materials better to adapt to the root canal and post configuration. Once the resin system which forms semi-IPN polymer has been initiated, the reaction time to reach the final degree of monomer conversion is slightly longer than with cross-linking monomer system of Bis-GMA only [38].

Clinically it is of importance to have the best possible post-curing adaptation of the cement and post to the walls of the root canal. This is important because the physical contact of the post and the cement by the adaptation forms the basis for the dentine bonding. It is also important to receive sealing against possible microbe penetration through the gaps towards periapical region of the restored teeth.

\section{CONCLUSION}

The results of the present in vitro study suggest, that by using an individually formed root canal post with direct cementation technique, less gap formation may be found between the cement and root canal dentine.

\section{CONFLICT OF INTEREST}

The authors confirm that this article content has no conflicts of interest.

\section{ACKNOWLEDGEMENTS}

The authors express their thanks to Stick Tech Ltd, Turku, Finland for providing the fiber material for the study. The financial support by the Finska läkaresällskapet (grant of Linda Gadd) is greatly appreciated.

\section{REFERENCES}

[1] Duret B, Reynaud M, Duret F. A new concept of corono-radicular reconstruction: the Composipost (2). Chir Dent Fr 1990; 60: 69-77.

[2] Sewón LA, Ampula L, Vallittu PK. Rehabilitation of a periodontal patient with rapidly progressing marginal alveolar bone loss: 1-year follow-up. J Clin Periodontol 2000; 27: 615-9.

[3] Pegoretti A, Fambri L, Zappini G, Bianchetti M. Finite element analysis of a glass fibre reinforced composite endodontic post. Biomaterials 2002; 23: 2667-82.

[4] Vallittu PK. Survival rates of resin-bonded, glass fiber-reinforced composite fixed partial dentures with a mean follow-up of 42 months: a pilot study. J Prosthet Dent 2004; 91: 241-6.

[5] Kargül B, Caglar E, Kabalay U. Glass fiber-reinforced composite resin as fixed space maintainers in children: 12-month clinical follow-up. J Dent Child 2005; 72: 109-12.

[6] Meiers JC, Freilich MA. Design and use of a prefabricated fiberreinforced composite substructure for the chairside replacement of missing premolars. Quintessence Int 2006; 37: 449-54.

[7] Schmitter M, Rammelsberg P, Gabbert O, Ohlmann B. Influence of clinical baseline findings on the survival of 2 post systems: a randomized clinical trial. Int J Prosthodont 2007; 20: 173-8.

[8] Ferrari M, Vichi A, García-Godoy F. Clinical evaluation of fiberreinforced epoxy resin posts and cast post and cores. Am J Dent 2000; 13: 15B-8.
[9] Fokkinga WA, Kreulen CM, Vallittu PK, Creugers NHJ. A structured analysis of in vitro failure loads and failure modes of fiber, metal and ceramic post-and-core systems. Int J Prosthodont 2004; 17: 476-82.

[10] Peroz I, Blankenstein F, Lange K-P, Naumann M. Restoring endodontically treated teeth with posts and cores-A review. Quintessence Int 2005; 36: 737-46.

[11] Lassila LVJ, Tanner J, Le Bell A-M, Narva K, Vallittu PK. Flexural properties of fiber reinforced root canal posts. Dent Mater 2004; 20: 29-36.

[12] Bitter K, Noetzel, Neumann K, Kielbassa AM. Effect of silanization on bond strengths of fiber posts to various resin cements. Quintessence Int 2007; 38: 121-8.

[13] Le Bell-Rönnlöf A-M, Lassila LVJ, Kangasniemi I, Vallittu PK. Load-bearing capacity of human incisor restored with various fiberreinforced composite posts. Dent Mater 2011; 27: e107-5.

[14] Le Bell A-M, Tanner J, Lassila LVJ, Kangasniemi I, Vallittu PK. Depth of light-initiated polymerization of glass fiber reinforced composite in a simulated root canal. Int J Prosthodont 2003; 16: 403-8.

[15] Mannocci F, Sheriff M, Watson TF, Vallittu PK. Penetration of bonding resins into fibre-reinforced composite posts: a confocal microscopic study. Int Endod J 2005; 38: 46-51.

[16] Sperling LH. Interpenetrating polymer networks: an overview. In: Klempner D, Sperling LH, Utracki LA, Eds. Interpenetrating Polymer Networks. $1^{\text {st }}$ ed. Washington: Am Chem Soc 1994; vol. 356: pp. 3-39.

[17] Vallittu PK. Interpenetrating polymer networks (IPNs) in dental polymers and composites. In: Matinlinna JP, Mittal KL, Eds. Adhesion aspects in dentistry. Leiden: VSP 2009; pp. 63-74.

[18] Le Bell-Rönnlöf A-M. Fibre-reinforced composites as root canal posts. PhD dissertation, Finland: Dept. of Biomaterials Science, University of Turku 2007.

[19] Torbjörner A, Fransson B. A literature review on the prosthetic treatment of structurally compromised teeth. Int J Prosthodont 2004; 17: 369-76.

[20] Mannocci F, Sheriff M, Watson TF, Vallittu PK. Penetration of bonding resins into fibre-reinforced composite posts: a confocal microscopic study. Int Endodont J 2005; 38: 46-51.

[21] Vallittu PK, Ruyter IE. The swelling phenomenon of acrylic resin polymer teeth at the interface with denture base polymers. J Prosthet Dent 1997; 78: 194-9.

[22] Lastumäki T, Lassila LVJ, Vallittu PK. The semi-interpenetrating polymer network matrix of fiber-reinforced composite and its effect on the surface adhesive properties. J Mater Sci: Mater Med 2003; 14: 803-9.

[23] Le Bell A-M, Tanner J, Lassila LVJ, Kangasniemi I, Vallittu PK. Bonding of composite resin luting cement to fiber-reinforced composite root canal posts. J Adh Dent 2004; 6: 319-25.

[24] Le Bell A-M, Lassila LVJ, Kangasniemi I, Vallittu PK. Bonding of fibre-reinforced composite post to root canal dentin. J Dent 2005; 33: 533-9.

[25] Vallittu PK. Fibre-reinforced composites in root canal anchoring: mechanical requirements, structure and properties of the fibrereinforced composite. Int Dent S Afr 2006; 8: 20-7.

[26] Vallittu PK. Interpenetrating polymer networks (IPNs) in dental polymers and composites. J Adh Sci Technol 2009; 23: 961-72.

[27] Alander P, Lassila LVJ, Vallittu PK. The span length and crosssectional design affect values of strength. Dent Mater 2005; 21 : 347-53.

[28] Tezvergil A, Lassila LVJ, Vallittu PK. The effect of fiber orientation on the thermal expansion coefficients of the fiber reinforced composites. Dent Mater 2003; 19: 471-7.

[29] Anttila E, Krintilä O, Laurila T, Lassila LVJ, Vallittu PK, Hernberg R. Polymerization shrinkage and hygroscopic expansion of fiber reinforced composite using fiber Bragg grating sensors. Dent Mater 2008; 24: 1720-7.

[30] Tezvergil A, Lassila LVJ, Vallittu PK. The effect of fiber orientation on the polymerization shrinkage strain of fiber reinforced composite. Dent Mater 2006; 22: 610-6.

[31] Garoushi SK, Vallittu PK, Watts DC, Lassila LVJ. Polymerization shrinkage of experimental short glass fiber reinforced composite with semi-interpenetrating polymer network matrix. Dent Mater 2008; 24(2): 211-5.

[32] Feilzer AJ, De Gee AL, Davidson CL. Setting stress in composite resin in relation to configuration of the restoration. J Dent Res 1987; 66: 1636-9. 
[33] De la Macorra JC, Gomez-Fernandez S. Quantification of the configuration factor in Class I and Class II cavities and simulated cervical erosions. Eur J Prosthodont Restor Dent 1996; 4: 29-33.

[34] Tay F, Lousbinen RJ, Lambrechts P, Weller RN, Pashley DH. Geometric factor affecting dentine bonding in root canals: a theorethical modelling approach. J Endodont 2005; 31: 564-88.

[35] Lehtinen J, Laurila T, Lassila LVJ, et al. Optical characterization of bisphenol-A-glycidyldimethacrylate-triethylene glycoldimethacryalate monomers and copolymers. Dent Mater 2008; 24(10): 1324-8.

[36] Bouillaguet S, Schutt A, Alander P, et al. Hydrothermal and mechanical stresses degarde fiber-matrix interfacial bond strength in dental fiber-reinforced composites. J Biomed Mater Res Part B Appl Biomater 2006; 76: 98-105.

[37] Pastila P, Lassila LVJ, Jokinen M, Vuorinen J, Vallittu PK, Mäntylä $\mathrm{T}$. Effect of short-term water storage on the elsatic properties of some dental restorative materials: an ultrasound spectroscopy study. Dent Mater 2007; 23(7): 878-84.

[38] Viljanen EK, Skrifvars M, Vallittu PK. Dendrimer/methyl methacrylate copolymers: residual methyl methacrylate and degree of conversion. J Biomater Sci Polym Ed. 2005; 16: 1219-31.

Received: January 12, 2013

(C) Makarewicz et al.; Licensee Bentham Open.

This is an open access article licensed under the terms of the Creative Commons Attribution Non-Commercial License (http://creativecommons.org/licenses/by-nc/3.0/) which permits unrestricted, non-commercial use, distribution and reproduction in any medium, provided the work is properly cited. 